\title{
American University of Beirut
}

\section{Arne Dietrich}

\section{You're gonna need a bigger boat}

\begin{abstract}
:
Creative ideas, even complex ones, can be assembled unconsciously and enter the conscious mind in a fully finished form. But there is nothing mystical or sacrosanct about the mechanisms giving rise to this mental phenomenon. Progress on the neuroscience of creativity can only occur if we fully expose Cartesian muses for what they are: teddy bears for grownups. This is not to say that neuroscientists understand yet what happens in the innards of the machine when we are creative, but I hope to show here where we are and where we are going on this matter. The aim of this article is not so much to persuade readers of a naturalistic explanation of creativity but to help with conceivability; that is, help them envision what a scientific account could, possibly, look like. Without a path towards such an ersatz explanation, we have little hope to lift the dense fog enveloping our beliefs in the specialness of the creative process, the instinctive tug we feel that the creative spark comes from on high. This article plants a seed of how such a seemingly magical event can be reverse engineered.
\end{abstract}

\section{Biographical note:}

Arne Dietrich is Professor of Psychology, American University of Beirut, Lebanon. He holds a PhD in cognitive neuroscience from the University of Georgia, USA, and his research focuses on the higher cognitive functions of the prefrontal cortex, especially (1) creativity, (2) altered states of consciousness, and (3) the psychol-ogical effects of exercise. His major publications include a theoretical framework for the neural basis of creativity (Psychonomic Bulletin \& Review 2004), a review article of neuroscientific studies of creativity (Psychological Bulletin 2010), a new, mechanistic theory of altered states of consciousness, the transient hypofrontality theory (Consciousness and Cognition 2003, 2004) and the proposal of new explanations for the effects of exercise on emotion and cognition. He is also the author of a textbook on consciousness (Macmillan 2007). Professor Dietrich has given numerous invited lectures around the world and his work has been featured prominently in the international press. E-mail: arne.dietrich@aub.edu.1b

Keywords:

$$
\begin{aligned}
& \text { Brain - Creativity - Incubation - Insight }- \text { Neuroscience } \\
& \text { TEXT Special Issue: Creativity: Cognitive, Social and Cultural Perspectives } \\
& \text { eds. Nigel McLoughlin \& Donna Lee Brien, April } 2012
\end{aligned}
$$


Even for the wilderness of human thinking, creative ideas seem to be deliberately designed to defy empirical enquiry. There is something elusive and mystical, perhaps even sacred, about them, isn't there? They come as they please and there seems little you can do to force their appearance. Lucky those who have them. No wonder humans have always mystified creative ideas - visits from the muse or light bulbs in the head come to mind. Anecdotal stories abound that portray the creative process as effortless and unintentional. From Kekulé's (1890) daydream of whirling snakes forming a (benzene) ring to Coleridge's (1816) poem 'Kublai Khan', such flashes of insight are the very cliché of creative genius. We are enamored with this romantic view of creativity. But, of course, there is nothing mystical about these kinds of thoughts. Unless you are away with the pixies, or fall for Cartesian ghosts hiding in dopamine synapses, you must concede that creative ideas are, like all mental processes, mechanical beasts that are computed - yes, computed - in the 3-pound, mushy pile of electrified biochemistry that is your brain.

\section{Did you really think you could sail the seven seas in a little rowboat?}

This leaves us with a lot of irritating questions. Just where do these creative insights come from? And, how do they rise from the depths of the unconscious to dominate the mind the way only food, gold or sex can? Psychologists and neuroscientists who have gone fishing for these slippery creatures in the muddy waters of the brain's hinterland must have known that there are easier career paths. But, beyond the obvious lofty, humanistic goal of understanding a defining characteristic of the human condition, there are also practical reasons for pursuing such a research program. Creativity is the fountainhead of human civilizations. All progress and innovation depend on our ability to change existing thinking patterns, break with the present and build something new. Think about it: if we can identify the basic principles of how, exactly, brains manage to be so ingenious, we could tinker with the process - and enhance it - with potentially enormous benefits for society. This prospect is not farfetched, although it does look, at present, far off. Despite the central importance of this most extraordinary capacity of the human mind, the few trailblazing scientists who have taken up the tricky hunt over the past decades have had their theories shipwrecked on the hard, rocky cliffs of reality. Consider the stunted idea of creativity being in the right brain. Honestly, could we really hope that such a simplistic construction holds water (Arden et al. 2010, Katz 1997)? The tsunami-like tidal wave of modern neuroscience sunk that little boat as swiftly as it did all the other floating death traps such as divergent thinking, defocused attention, lateral thinking, unconscious processes, altered states of consciousness and, related ideas such as bipolar disorder and autistic savants (Dietrich \& Kanso 2010). What has become apparent in recent years is that creativity is too complex, and too distributed in the brain, to be captured in a net held together by such premature category formations and ontological danglers. How else, then, do you tackle a problem that size? We could start by taking the famous advice Brody gave Quint in the movie Jaws after getting a first glimpse of the size of the great white: 'You're gonna need a bigger boat.' 


\section{What we should expect to see, based on what we know}

Modern brain science is on a roll. You have surely heard of these snazzy new neuroimaging gizmos that can take a snapshot of your mind in 256 million colors while you ponder a Buddhist koan. Add to this a fearless army of cognitive psychologists devising ever more sophisticated experiments and you have a veritable explosion of new knowledge in this area (for an introduction, see Gazzaniga et al. 2009). Cognitive neuroscience now possesses the theoretical scaffolds it needs to build the kind of boats that can catch creative ideas. The brain no longer is the inscrutable machinery it once was.

It is my hope here to bring you a sense of where we stand on the vexed problem of creativity and perhaps a sneak preview of where our on-going voyage of discovery seems to be heading. The main planks of my efforts require that you become a bit more fluent in brain-speak. But don't fret; this article is for the non-specialist. It also won't give the appearance of being committee-written, seemingly emanating from noperson, arguing for or against some obscure position, only to conclude with the need for more research. Instead, I'll take an unusual vector. Based on what we know, which isn't much but probably more than you'd expect, I intend to tell the story of how creative insights come, in all likelihood, into existence. But with so many bits of the storyline still shrouded in dense fog, I am forced to embellish the facts with some fiction. So be forewarned. What follows is two parts popular science and one part educated guess work. You do need to bring a bit of steadfastness to this sci-fi tinged adventure, though. A mechanistic explanation of creativity comes with hardcore materialism, a position that tends to give people existential vertigo, an understandable reaction, really, considering that what is at stake here is the nature of the soul. When sailing so close to the wind, it is never easy to ignore the sirens of dualism that lure us with their enchanting songs to false islands where clever homunculi fix all remaining problems. Another danger lurks behind the myopic theorizing of modern-day phrenologists. Don't pay money for old rope when they proclaim that all is explained by increased neural activity in some unpronounceable part of the brain's cortex (Dietrich 2007b). No one likes the feeling of sailing off the edge of their intellectual comfort zone into unknown regions where monsters like Uncertainty live. Yet, we must if we want some real answers - sound mechanisms, that is.

\section{Aground, waiting for the tide}

The brain is a wonderful thing. It starts working the instant you're born and never stops until you must write something creative on deadline. So what do you do when you experience writer's block, when you are totally stuck staring at the blank page on your computer screen? If you are like most people you try to break the impasse by removing the problem from the limelight of consciousness - a stroll in the woods perhaps. And, all of a sudden, as if from on high, inspiration hits and your unsuspecting conscious mind is the lucky recipient of a brilliant solution. You run to your computer and write the essay, start-to-finish, in two hours. Psychologists call this 
trick incubation (Runco 2004), and there is universal agreement that it works. But how?

\section{Descartes' ghost ship: Decommissioned but not yet scrapped}

Clearly, it is one thing to commit yourself to a materialist view, it is quite another to accept all the inevitable consequences such a view brings with it. For a thoroughgoing, uncompromising theory of mental life, we have to let go of more of our cherished intuitions, however. The one intuition we must abandon, above all else, goes something like this: I experience myself as being somewhere inside my own head. I look out at the world through the peepholes that are my eyes, catching the sights that are out there. Information enters through my senses and is built up, by my brain, into mental representations that are then displayed on a virtual mental screen or some kind of theatrical stage, for me, the audience, to see and appreciate. From my little cranial command post, the place inside where 'I' am, I can order my brain to move my body. So put, and unduly sharpened, this is how having a mind feels to us, isn't it? This story implies, however, that there is a special place - a stage or screen that is brightly lit, where information is displayed for the Mind to be acted upon. Problem is, none of this exists. There is no central place anywhere in the brain; no show reeling off specially selected conscious information; and no Higher Executive who inhabits the brain and to whom consciousness, or creativity, happens.

\section{Setting sail without homunculi}

Neuroscientists universally agree that there is no such pivotal place where it all comes together (Baars 1988). Nor is there a Central Experiencer, an Ego or Self, separate from the hubbub in the brain. The brain is a massive, parallel-processing system. Its activity is distributed in multiple, independent streams of processing that are, at no time, synthesized in one central location into a coherent image for the mind's eye. To see the predicament of this theoretical dud in full, consider this. Once we enter this so-called Cartesian theatre (Dennett 1991) and re-represent the data that has been already completely analyzed, we must also conjure up a fully conscious homunculus, a little man - or muse - inside the head, who marvels at the show, controls the consoles and gauges, and ultimately pulls the strings. But then we are stuck explaining the inner workings of this homunculus and an infinite regress immediately looms. But worse, the homunculus - conveniently enough - just happens to have the right kind of powers and abilities to explain what needs to be explained in the first place. The homunculus, then, does the very job that a theory of mind is supposed to do. This is a pernicious trap! We make some progress for a while, explaining this and that aspect of creative thinking, and right when the going gets tough we flee into comforting realms of miracles by inventing - sim sam sum - an internal agent - our muse - who handles all the bits still missing. The trouble is, this lures us into thinking that we've done the job, that we can stop, when, in fact, we have solved nothing. All we've done is kick the can down the road. We are no better off resorting to miracles on the tenth step than - like Descartes - on the first. Once the ghost is in the machine, 
you are tangled up in the cul-de-sac of dualism. We would have succumbed to the powerful seduction that there must be some other, further place where the mind is. No doubt, this intuition is brutally difficult to shake. But we have no choice. The best thing to do when you catch yourself relapsing into this kind of medieval thinking is to follow the materialist's two-step program. First, remind yourself that, at a minimum, your private experience of your own mind is factually mistaken. Second, ask yourself the logical next question: What, exactly, is it you are pointing to? Who is this 'me' reading the neurological record? What you will find is not some Higher, All-Knowing CEO, but something that, upon further inspection, can itself be broken down into subcomponents and embedded into the operation of the system.

The homuncularism here is stopped the way an infinite regress is usually stopped in philosophy - right at the beginning. The infinite regress is defeated in two easy steps. First, we break down consciousness and distribute it throughout the system right at the outset. By embedding experience into the operations of the brain, we avoid building up a big mystery that, eventually, can only be upheld by invoking some type of divine intervention. Second, we make higher homunculi less conscious than their hosts and the homuncular regress becomes finite, bottoming out with homunculi so simple that we can replace the top ones with dumb neurons, each itself as unconscious as a silicon switch. For creativity, this inversion of thinking allows us to take the Designer out of the design process. Creativity cannot come from on high.

Naturally, this leaves open the burning question of how else we should think about the mind. How can we kick this misbegotten conception and really understand, at an intuitive level, what's going on? And that rather neatly takes us to the next topic.

\section{A ragtag group of neurons surfing the stream of consciousness}

At this point, I hope you are prepared to flip your thinking. Nothing short of it will do, I am afraid. In what follows, we first acquaint ourselves with a few key concepts of how neurons go about doing their business. Neural networks in the brain aren't hardwired. Well, some are, especially in the lower brain, but many circuits develop as we go through life. Those neuronal circuits contributing useful information processing are strengthened while those that don't wither away. The whole brain develops and operates through competitive interactions among neuronal populations (Crick \& Koch 1998). Now, underline the word competitive here.

Like all complex systems, the brain has a unique problem. As said, it is a massive, parallel-processing system in which countless specialized and independent modules have evolved to handle very specific tasks. You have to wrap your head around this, but like it or not, your brain isn't a single, monolithic and unified entity but it is rather a diverse society which comprises umpteen individual member neurons. What's more, each is trying to beat the other at the same game, that is, to gain access either to consciousness or the right to move muscles. Actually, the former, access to consciousness, is just a way to get to the latter, to determine the organism's next move. Here, then, is the unique problem. Clearly, thoughts and movement are serial phenomena. We don't have two streams of consciousness or make two separate 
movements to lift a cup of coffee. We experience the world, including ourselves, as an integrated whole and not composed of bits and pieces. But why, given the many independent agents? How does a superduper complex, multi-unit system come up, in a reasonable amount of time, with a single decision?

The notion of cerebral anarchy is initially a bit counterintuitive, not to mention discomforting, but it grows on you once you see how it enlightens some otherwise puzzling phenomena. The basic idea was first mooted by Oliver Selfridge in his 1959 pandemonium model, which he devised to solve exactly that problem. The pandemonium model was a computer program in which hordes of independent demons are locked into an internal struggle for supremacy. But in the end they selforganize, bottom-up, and manage to put out a single solution.

\section{A comparison of two forms of government}

A metaphor neuroscientists like to use to get the same idea across is to liken the brain's internal dynamics to a parliamentary democracy (Crick \& Koch 1998). To gain access to power, individuals in a democracy must garner support for their cause. So they form parties and create broad coalitions. A winning coalition eventually emerges and forms, temporarily, the next government that determines a whole country's course of action. It's essentially a single output decision from a society of millions of individual units, each with its own mind and its own idea of what to do. In principle, the case is the same for neurons, except that the brain probably isn't so much a democracy with orderly elections as it is a sort of gang war ruled by law of the street.

Like people, neurons group together flexibly into clusters and assemblies because they, too, are profusely interconnected in sets of networks. To make their computation felt in consciousness, each coalition must campaign for a larger support base. By recruiting other neurons along the way, they form transient super-coalitions to better compete against other strong neuronal assemblies. There is no need to get all technical about it, but several physiological parameters are involved in building the internal strength of such coalitions - number of neurons, temporal synchronization of neuronal firing patterns, mutual entrainment or phase locking, to give a few prominent ideas. The top dog is ultimately determined on the basis of a competitive process, with the victorious coalition, the one with the largest backing, having its computation sustained long enough to either become conscious or contribute directly to the organism's next move. To put it another way, we settle on a particular output on the basis of a head-to-head competition. Any conflict between parties is resolved in playoff fashion with a winner-takes-it-all strategy that leaves no room for a loser or powerful semifinalist that might compromise purposeful movement or the single stream of consciousness. The unity of consciousness is achieved by way of an atrocious elimination tournament that sees only the last man standing - actually not much of a democracy, come to think of it.

Note how this concept of competing cell-assemblies does away with the homunculus, the infinite regress of an entity watching the happenings in the brain, because the 
dynamics are governed by a bottom-up process (Churchland 2002). Although the competition is likely to be strongly biased by attention, via a top-down process (Dehaene \& Naccache 2001), attention is not meant to substitute for some kind of Cartesian King appointing the next government. The cranial Supervisor itself dissolves into functions that themselves need no supervision. Remember the two-step program.

\section{5 minutes}

This view demands from us that we think of the brain as we do of a complex society, a loose federation of independently operating specialist modules with no dominant center or master homunculus. There are, of course, higher-order neurons providing some integration but there is nothing inherently special about them, functionally or structurally. Nor do they peak out at some organizational summit where consciousness resides. Naturally, there is also no ' $\mathrm{I}$ ' doing the viewing or making the decision; this illusion arises simply from the self-organizing functional properties of the system, in which some information asserts more or less control over behavior. In the brain, conscious information processing is a moving target, a shifting supercluster of strongly interacting neuronal populations (Dehaene \& Naccache 2001).

If you have problems visualizing this, you are not alone. Perhaps we can try a different crutch for the imagination. Think of consciousness as fame in the brain, or cerebral celebrity. When some information in the brain becomes conscious, it is famous in the sense that it is widely known among other demons in the brain (Dennett 1991). Once brightly lit, famous demons assert a greater influence over how the system, as a whole, functions. Like celebrity status in society, selection to stardom does not require a higher power, but is simply the result of a competitive, bottom-up process. The losers of the competition, the coalitions of demons that for some reason could not muster up enough clout, dissipate into oblivion never to get close to a motor neuron. Note that fame does not lead to, or is separate from, consciousness; it is the property of having influence.

The stream of consciousness, then, is nothing but a special kind of illusion (Blackmore 1999). It is a necessary illusion. Imagine we'd have intimate access to all the behind-the-scenes mayhem of the brain. We'd go mad! What we do is to simply probe this multitrack system at different times and places and filter the underlying mentalese into a narrative stream that acts as a decent indicator of what the organism, as a whole, is doing. The content of consciousness, creative ideas included, is less like a continuous, integrated stream than it is a series of discrete contents, sequenced together on the fly from different parallel streams, each consisting of continuously shifting coalitions of neurons. From this emerges, at the macro level, the story we tell ourselves, though this is not what's really going on underneath. The mind is a sort of 'benign user illusion' (Dennett 1991: 311) of the vastly complex brain similar to an operating system like Windows, which is a user-friendly version of the vastly more complex computer. It streamlines the myriad of parallel neural processes into a stepby-step narrative that makes the brain seem to work as if it were a serial processor. 
The upshot is that we can tell others a coherent story, a quick summary of what is going on, at the level of the whole organism.

Like fame, conscious information is information that is globally accessible, which helps it spread fast through a very large number of autonomous, individual processors. Going global this way is simply the best way to facilitate communication in a massively parallel-processing system that needs to make a single, purposeful move in real time (Baars 1988).

\section{How one thing leads to another}

What did you do on April 18, 2007? How would you go about looking for this information in the convoluted vaults of your cerebral cortex? Knowledge representation, it is agreed, organizes information in clusters or categories connected by associations that link items either in time, space, or semantic meaning (Anderson 1996). Our general knowledge is not organized like a database that can be searched with different keywords from different angles. This can be easily seen by the difficulty of retrieving information that cuts across clusters or categories, for instance, recalling the months of the year in alphabetical order or listing all the animals that are green. Network models emphasize interrelation between items.

Take the best-known one, the semantic network model (Collins \& Loftus 1975), which is based on the idea that knowledge consists of concepts that are interconnected in a network-like structure. The model represents concepts as nodes, which are linked to each other in a way that takes into account the strength of their associations. When we retrieve information, or activate a concept, the activation will spread to other concepts and eventually throughout the network. This process, appropriately called spreading activation in the trade, proceeds by favoring a path along the strongest semantic links - like lightning following the path of least resistance. Naturally, the strength of any connection depends on a person's particular life story. Another way of saying this is that the nodes of the knowledge structure have weights that can shift and come to reflect over time our biases, preferences, and experiences. Now, hold that thought; we will come back to flexible weights when we get to creative ideas generation.

\section{A little detour into the machine's innards}

A quick refresher of a basic memory subdivision is useful at this point to prevent conceptual howlers once we push for a mechanical model of creativity. In considering memory systems, you are reminded that many complications remain clamped to keep in focus the points pursued here. The explicit memory system (a separate, implicit system which we ignore at this point also exists, see Reber 1993) has two main components, working memory and long-term memory (Tulving 1995). Working memory describes the ability to process information online. It is a monitoring system of on-going events that temporarily keeps in mind knowledge that is relevant to the situation, so that one can 'work' with it (Baddeley 2000). It is in working memory 
that you are holding the information you read in the beginning of this sentence so that the end of this sentence still makes sense to you. Also accepted is the view that the information temporarily buffered in working memory is the content of consciousness (Dietrich 2003). This is not to say that working memory is the seat of consciousness but that information must be represented there to be fully conscious, or if you prefer, globally accessible and famous. This is important for us because creative insights, by definition, occur in consciousness. In other words, for information to shift in status from unconscious to conscious it must find, one way or another, its way from the illlit basement in long-term memory into the brilliantly illuminated buffer of working memory.

\section{Up, up and away}

In planking our boat, we help ourselves to the very useful concept of a task set (Allport 1987, Monsell 2003). Scientists explain all sorts of interesting effects with it that pop up whenever people in a psychology lab switch from one mental task to another. As an example, take cooking pasta, presumably a straightforward affair. Like most things you handle every day in cruise-control mode, you probably think there's nothing to it. But the complexity of pasta making would quickly dawn on you if you had to explain it to someone who has never cooked. Better yet, imagine programming a robot to do it. Brains are commonsense lubricated. But for robots, every element of a task must be specified, even the force vector of a single joint in one finger of the hand that is to grip the salt shaker, to say nothing of lifting it, positioning it over the boiling water, turning it over, or a million other bits of implicit knowledge.

Done with one task, you turn your attention to one of life's next little challenges, a task sure to be as complex in its sub-components and number of sub-routines. Naturally, each task calls for specific types of skills. In response, brains don't simply make mental resources available in a generic sort of way but configure them to suit the task's unique set of requirements (Monsell 2003). That means that it must also reconfigure these resources when switching to the next task.

The phrase 'task set', then, denotes the configuration of mental resources that goes with a task (Allport 1987). For our intents and purposes, we can also use the term 'mindset' to mean the same thing (although mindset is broader and independent of a specific task, as in 'I am conservative and vote Republican'). At the conceptual level, the idea refers to all the operational parameters the neural network is set to - initial default settings of the nodes (the influence a node asserts in the knowledge structure), control processes, permissible transformations, category boundaries, expectations, and troubleshooting instructions. Task sets are specified to astonishing detail, like how long it should take for the elevator to move after you press the button or what shape a cake would have after you bite off a piece, a matter of some social importance if you balance it in your hand. A task set is a kind of mental schema containing the elements, and their values, that are tagged as temporarily belonging together in the network because they played a role in completing the task in the past. Violating the network's 
predictions, as you can imagine, is a key event - perhaps even a necessary one - that starts the search for insight solutions.

\section{Beginning, middle but no end}

You cannot perform a task until the cognitive system is properly arranged and organized. Like a tabula-rasa robot, you wouldn't have a clue about how to approach the task. Should the task change, the task set that goes with it must first be uploaded, so to speak. Task-set reconfiguration costs time and resources because a new task triggers the activation of the whole task set that is associated with it (Monsell 2003). All knowledge nodes that are part of the new neuronal coalition must shift their weights and the strength with which their connections operate must be recalibrated. The upshot is of course that, so-primed, the spreading activation of information in the neuronal coalition is biased in such a way that allows the network to respond efficiently to whatever the task throws at it. These activity patterns are stored as whole entities in memory. When you prepare to drive your car, the network is specifically enabled for that very task, as opposed to, say, playing tennis or ironing a shirt.

The need to switch task sets can be forced by a situation demanding attention - the smell of chocolate, Angelina Jolie walking by, that sort of thing. Some stimuli so strongly activate a task set that the tendency to perform the task is irresistible and unintentional. Reading labels on cereal boxes during breakfast is a good example. A task set turnover can also occur because you decide to shift your focus, or more likely, because you cannot uphold the current task set for some reason. As a friendly reminder at this point, beware of the sirens of dualism here. This 'you' does not ride above the fray. It is also a broad coalition of neurons, higher-order but less conscious, which simply help nudge the current government from power. There is, turns out, an apt name for these influences of volitional control; they are called top-down projections in neuroscience. In either case, the change in mindset ripples through the network, altering coalition membership and weight configurations, which results in the re-setting of the whole cognitive system.

A mindset can be activated more or less strongly. The brain constantly performs a tricky balancing act to guarantee effective cognition. On the one hand, a task set must have some internal stability to keep the ongoing task from interference and disruption by other task sets. At the same time, the activation must allow enough flexibility for mental gear changing so that it can adjust if the context necessitates it. Much effort goes into this evolutionary calculus of trade-offs between stability and flexibility; weak task sets cannot stay in power, strong ones cannot be overthrown.

Many brain scientists think that the main effort of the reigning task set, apart from grappling with the actual task, goes into keeping its ruling coalition together and solidifying its power base. In the same way a country's president or prime minister devotes seemingly endless amounts of time to dealing with the opposition or campaigning for re-election, task sets must, in good Darwinian tradition, strongly inhibit other, competing task sets if they want to stay in power. Failing to suppress 
hopeful contenders leads to a fall from the stream of consciousness. Phenomenological content would then be whatever it is the new ruling neuronal assembly is computing. One reason for the high cost of inhibition stems from the fact that networks are not discrete entities. Knowledge nodes can simply shift allegiance and help send the ongoing spreading activation down a different path.

\section{Surprising adventures in the far reaches of consciousness}

So what happens when, for any number of reasons, the motley crew of neurons currently calling the shots in your conscious mind destabilizes? Three options present themselves. One is that a new and powerful federation of brain cells is waiting in the wings. Take the task set right now ruling your mind - reading, presumably. What chance does it have against the brain circuits that are activated should your blood sugar levels nosedive? Option two involves bailing out the teetering government. Remember these top-down influences on the knowledge structure I was on about earlier. The pandemonium idea is not just a grassroots model. Of course you can also stay on task by the sheer force of concentration. Focusing attention to the task has the effect of rigging the competition among neurons at the base. Top-down amplification boosts the weights of some nodes at the expense of others; it is the mind's equivalence of a powerbroker, a large cash donation to a politician's re-election campaign. It keeps the incumbent dominant in working memory by simply overwhelming the resources that other candidates possess.

Matters take a different - and highly fascinating - turn with option three. As every schoolchild knows, paying attention has limits, especially as time goes on. Ever tried sitting through a five-hour Wagner opera? Suppose the leading alliance disintegrates all on its own. Perhaps it cannot maintain the internal strength among its loose connections and simply self-destructs. Or perhaps the costs of running the strong inhibitory control processes become prohibitive. Suppose further there isn't a willing, or able, replacement standing by, ready to fill the impending power vacuum - think fall of Rome, not Russian Revolution. The mind state that follows must rank among the most fun things to do in life. I am of course talking about the ephemeral and aimless drifting otherwise known as daydreaming. Without a dominant force ruling the phenomenal world, conscious thinking careens pinball-style through one imaginary scenario after another.

You will remember that we got into the finer points of governmental politics in the first place to highlight the role of incubation in the formation of creative ideas. So, irrespective of which of the three options transpires - coup d'état, dictatorship, or mindwandering - the million-dollar question as to how incubation facilitates insights is this. What happens to the old task set?

\section{Crossing into uncharted territory}

It is probably not too much to say that from here on forward our journey effectively turns, due to the lack of hard data, into a mini thought experiment. There are endless 
difficulties attached to telling the story right, which force us to occasionally rise to levels of speculation most paying members of the Society for Neuroscience would safely call imprudent. But this being an essay on creativity in a creative writing journal, there is a lot we can do - a few leaps of faith here, some embellishment there - if we adopt an elastic take on the scanty evidence there is. So, without the neckbreaking mental gymnastics one must perform these days when trying to understand the contemporary cognitive science literature, and the encrypted neurolingo in which it is shrouded, here are the few possible answers to the above query that have so far escaped the dustbin of wishful thinking.

Possibility one is the befittingly-named task-set inertia (TSI) (Allport 1987). Bits of the TSI story are known, many details are not, making flag-waving simplifications of what is known unavoidable. Briefly (and roughly), brain circuits are not binary on/off, yes/no switches. A strongly interacting assembly of neurons that has just fallen from grace surely needs a while to return to baseline. Like everything else going at full throttle, that should consume time, time during which the coalition would still reverberate together with the information that was whirling around its network before. The critical difference is that the neural activation would now hover at intensity levels below the threshold of consciousness. There is no principled reason to assume that expulsion from the working memory buffer spells instant death for a coalition. The evidence for such network aftershocks isn't exactly deep, wide, and thick (Waszak 2003) but there is enough of it that, in conjunction with common sense, we can assume that it is happening. Discussion in the literature on how strong this inertia might be or how long it could last is curiously ignored, like one would a tic. But this is a matter of obvious relevance, one would think, for coming to terms with creative insights. It is conceivable of course that a faltering coalition regains, all by itself, control of the throne by, for instance, recombining bits of the information during TSI in such a way as to find a solution to the problem. Hold that thought for a bit.

But of course it is never going to be as simple as that. The inertia idea relies solely on residual hubbub in the knowledge structure. It's the skinniest of all theoretical models and there are two considerations that suggest that a comprehensive explanation needs more work. First, creative insights have a way of popping up weeks or months after you last toyed with the problem. It is hard to see how inertia could linger that long. Equally tricky is the notion that the goal - the fact that there's still a problem in need of a solution - is embedded at the level of the knowledge nodes. This sounds more like the handiwork of a higher-order brain circuit and probably is.

Possible answer number two, then, involves the idea of fringe working memory (Cowan 2001). Like task-set inertia, the name describes the core idea aptly. It implies that working memory has a focal center and a fringe with the latter buffering knowledge that still has some conscious aspects and/or is relegated to a lower priority. Illustrating the fringe best perhaps is the annoying metacognitive knowledge that is the hallmark of the tip-of-tongue experience. You know that you know but that clue alone cannot ignite the associated knowledge network (Koriat 2000). Fringe working memory lacks qualitative content; it only represents that something is represented, but not what that something is. An index, in other words. We are led to this conclusion by 
empirical work showing that goal pursuit, thought formerly only possible in consciousness, occurs also outside a person's awareness (Dijksterhuis \& Aarts 2010). Put another way, the goal of the problem now incubated remains active in fringe working memory and still organizes, from that position, the spreading activation in the knowledge structure below. Such a setup could also have the effect of prolonging the TSI.

\section{Between the devil and the deep blue sea}

It is hard to escape the thought that possibilities one and two have both a hand in the incubation effect. The trick might work like this. Conscious thinking also occurs at two levels, knowledge nodes reshuffling long-term memory bits and working memory boosting these combinatorial thought trials with the injection of higher mental faculties - self-reference, sustained attention, counterfactual thinking, goal representation, hypothesis testing, mental time travel, and so on. Why not presuppose that unconscious thinking operates in the same way, only with less oomph? While task set inertia keeps the underlying network primed, fringe-working memory remembers that there is still unfinished business out there. This active representation of a goal provides, via top-down projections, some organizational control that helps steer the spreading activation in the network towards a solution. A model combining both possibilities requires that the links between the two levels survive the dethroning from prime time.

But it doesn't end there. In the usually calm waters of academe, one more possibility has emerged as a bit of a hot-button topic. The sticking point is this. Some cognitive psychologists contend that for information to cross the threshold from a dormant, inactive to a conscious, active status, an additional process must enter the equation. You may have noticed that the above account makes do with only a single strengthening mechanism. What's more, it's also a linear one. A few (million) loosely aligned neurons form a coalition and start chatting together. Over time their bond grows stronger until they dominate the brain matrix, cross some sort of intensity threshold, and enter the global workspace and thus consciousness. There are good reasons to believe that such a simple, purely quantitative, story won't do. Some think that there must be a qualitative shift as well. Several options are on the table. One, for instance, involves the dopaminergic reward system (Schultz 2000). This would be an independent, secondary process that kicks in and it is perhaps that process that provides the signal to make the crossing between the unconscious and the conscious possible. An interesting advantage of such a setup is that a coalition too weak to enter the hall of fame on its own merit could be hived into power with the help of an influential friend. Of course, such a thing would never happen in real politics.

\section{Insights into insights}

By definition, creative insights occur in consciousness. Translated into brainspeak, this means that a new idea - regardless of where or how it was generated in your 
noggin - needs to ultimately find its way into the working memory buffer of the prefrontal cortex. This place is the final common launchpad of all creativity computed in the brain into the world at large. Only when posted there, on the blackboard of the global workspace for all the mind's demons to appreciate, is the idea famous and thus conscious. Although novel information can get to working memory in several ways, we can readily identify two general classes of mechanisms, the deliberate and the spontaneous mode (Dietrich 2004, 2007a). The concepts of top-down and bottom-up processes roughly map, respectively, to these two processing modes. While the deliberate mode involves effortful and voluntary retrieval of knowledge to derive new ideas; the spontaneous mode refers to the sudden and surprising representation of information in consciousness - hard thinking versus daydreaming, if you like. Allow me to drive home this point with all the force I can muster. Creativity can occur as a result of both modes, by systematic thinking as well as by careening through lala land. These processing modes differ in several important ways, which, incidentally, allow us to understand the incubation trick, but either/or does not enter the picture. Rather, both modes support different types of creativity.

\section{If at first you don't succeed}

It is obvious at a moment's reflection that creativity can be the result of laborious trial and error. What would we otherwise make of Edison's assembly-line approach to inventing, Watson and Crick's algorithmic style to decode the DNA, Bach's methodical way to compose hundreds of cantatas, the imaginative ways in which NASA engineers solved the problems of the otherwise doomed Apollo 11 mission, or the countless occasions each one of us converged on a creative solution by systematically eliminating alternative possibilities?

When we ponder problems in the deliberate mode, the sophisticated mental toolbox of the prefrontal cortex comes into play. These higher mental faculties enable us, among rather many other things, to focus our attention on the problem, deliberately retrieve pertinent long-term memories, and to flexibly recombine this knowledge in working memory. You could say that the prefrontal cortex has a kind of search engine that googles the brain's intranet and pulls into working memory task-relevant information (Cabeza \& Nyberg 2000). Once that knowledge is online - fully conscious - the whole arsenal of our higher cognitive functions can be brought to bear on the problem, yielding new ideational combinations of high complexity (Dietrich et al. 2001).

There is good reason to assume that the effortful retrieval of knowledge from longterm memory as well as the recombinatorial shuffling of that information in working memory is inherently structured. As part of the mental toolbox, the prefrontal cortex also houses a person's cultural values and belief system. It follows that its search engine, as it digs for relevant information in long-term memory, has a built-in predisposition; in orther words, the search itself as well as the recombination of informational units is constrained by a number of parameters, such as biases, expectancies, preferences, schemas and previous experiences. These psychological 
processes make a person's heuristic algorithms full of idiosyncratic fiddly bits (Dietrich 2004).

The upshot of such advanced heuristic search algorithms is, of course, speed. By preempting remote associations, counterintuitive paths or improbable explanations, the vast search space is trimmed down to a smaller region that is more likely to contain a solution. But therein also lies the drawback. These heuristic algorithms are predisposed to only retrieve knowledge consistent with a person's worldview and past experiences. That works well only if the solution is located within the currently prevailing paradigm. But what if the solution requires, to dust off this useful cliché, out-of-the-box thinking; what if it violates something you think is true about the world? Heuristics informed by rational thought and common sense are likely to consider such unconventional solutions to be remote associations and exclude those regions of the design space containing them. Think of it as the speed/nuttiness tradeoff. While the deliberate mode allows the thinker to direct cerebral capacities to the problem, it tends to check for the keys under the lantern only, where the light is. To quip, while heuristics have the advantage of limiting the solution space, they have the disadvantage of limiting the solution space!

\section{Rigging the competition}

Seen through the prism of neuroscience, the hallmark of the deliberate mode is the powerful presence of top-down influences biasing the competitive interactions between cell assemblies. Suppose you are presented with a puzzle problem. As an immediate response, you activate a task set by priming the underlying knowledge structure. That is, based on several parameters - past experiences, folk physics, folk psychology, etc - nodes in the network are calibrated so that the strength of their connections reflects the weight that each is predicted to contribute to the puzzle's solution. This is a node's individual baseline activity, its frequency of occurrence. This parameter, the frequency of occurrence, matters for the simple reason that the value it is set to for each node determines how specific information makes its rounds on that network. Let's say you have an idea how the puzzle might be solved and you run this option on the preset network structure. Based on the frequency of occurrence of all the nodes, this idea is then processed at particular speeds, which are likely to be different to the speeds occurring for other solution paths.

In the deliberate mode, the top-down input from prefrontal regions shifts the frequency-of-occurrence weights even further. Lowering the values of some nodes while increasing those of others is, in effect, how search heuristics are implemented at the level of the knowledge structure. Amplifying the existing task-set biases further creates even greater information-processing speed differentials for good as opposed to bad or unworkable solutions. It also makes the knowledge structure less flexible - or more streamlined, depending on your point of view - when it processes information. This is because stronger weight values, in either the plus or minus direction, provide less opportunity for spreading activation to go off on remote tangents. Such an 
opinionated knowledge structure is a highly efficient search engine but only, as said, if the solution conforms to predictions embodied by the weight assignment.

We can draw this out from yet another angle. Details aside, the creative process is, essentially, an evolutionary variation-selection algorithm. We generate new ideas and then test them on the basis of some fitness function - viability, functionality, symmetry, beauty, etc. While variation is naturally present during information processing, we can think of the speed of processing, as a selection process because this parameter is capable of identifying value or fit. This may sound trivial or too simplistic to some but, according to this view, whatever information is processed with greater speed has a higher fitness and is thus selected for. Speed of processing may well be the key strengthening mechanism that allows the neuronal coalition handling the information to gather enough support for the ascendency to consciousness.

\section{Incubate baby, incubate}

Suppose, however, the clever move that needs to be made is located in left field, in one of those regions of the search space excluded, a priori, by the strong directional guess of your heuristic algorithm. One sort of information falling under this category is scientific knowledge; for none of it makes any intuitive sense. No amount of logical maneuvering would have ever convinced some ancient seafaring Phoenician that the Earth is round, that it has a molten core, or that it is at the outer arm of some small, insignificant galaxy, to say nothing of eleven-dimensional string theory or, for that matter, cell phones. The fact that scientific advances are the upshot of the mind goofing off in imaginary dreamscapes should make us very cautious about crediting creativity solely to the higher cognitive functions of the cerebral cortex.

Don't underestimate creative achievements that buttress an established paradigm either. Think of all the great artwork of, say, the Renaissance, the Romantic Period, or Impressionism that relied on the ideals and techniques if its time. In general, great pieces of art that we, in retrospect, consider masterpieces were not those that opened new directions but those that emerged at the height of a paradigm. But if a creative product, in the arts or sciences, requires a paradigm shift for its successful wheeling, the deliberate mode is likely the wrong tool for the job. Since we don't know ahead of time which projects necessitate revolutionary thinking, we first have a go at a problem by systematically exploring our intellectual comfort zone. In the deliberate mode, however, this leads inevitably to frustration. After exhausting all options that seem logically or aesthetically possible, you will, at some point, give up.

\section{The world does not disappear when you close your eyes, does it?}

But that isn't to say that your brain has thrown in the towel just because some other information has staged a successful coup in the global workspace. By temporarily or permanently removing the issue from conscious awareness, the brain merely shifts the puzzle problem from a deliberate mode to a more spontaneous mode of processing. Thanks to task set inertia and fringe working memory, the puzzle doesn't just 
disappear, although that's what it feels like to us. It doesn't take a whole lot to start the incubation phase. As anyone who ever sat through a boring lecture can attest to, concentration has clear limits. During the inevitable times when the attentional system is downregulated - in daydreaming, for instance - thoughts unguided by sociocultural norms and unchecked by conventional wisdom become represented in working memory. In such a mental state, the content of consciousness is characterized by unsystematic drifting, rendering the sequence of thoughts manifesting itself in consciousness more chaotic (Dietrich 2004).

If you prefer to use computational terms, you could say that the brain now runs less restrictive - but also slower - heuristic algorithms to look for design improvements. By virtue of not being initiated in - and supervised by - the prefrontal cortex, the drawback of these bottom-up search functions is that they do not benefit from the generally excellent, and efficient, forecasting ability that can only come from higherorder thought. But what they lack in speed they make up for in nuttiness; for they can, now and then, chance upon smart moves that don't look so smart to your preset mental schemas.

\section{Like fish unaware of water}

Altered states of consciousness, of which daydreaming is a very mild sort, are accompanied by reduced activity in prefrontal cortices (Dietrich 2003). There is a technical term for this - hypofrontality. Incredibly, this hypofrontal effect of altered states sheds a great deal of light on some of the more peculiar phenomenological oddities characterizing the hallucination zone - the disintegration of the sense of self, the inability to sustain attention, or the feeling that time stands still (Dietrich 2007a). To stay with daydreaming, the downshifting of the prefrontal cortex into safe mode removes the - mostly inhibitory - top-down projection on the knowledge structure underneath. This, in turn, resets the network nodes to much less polarized frequencyof-occurrence values. It also puts an end to the highly selective retrieval of information and the highly restrictive way in which it can be recombined. The net result is that the network is much less biased in the way it conducts its business. The spontaneous mode is characterized by a more level playing field that gives a fair chance to the many different autonomous neuronal coalitions to compete for stardom. The spreading activation in such a neutrally calibrated network simply diffuses more readily, in all directions, and unconscious thinking is more hit and miss as a result.

Daydreaming is a mental state in which no powerful task set steers the ship - the fallof-Rome scenario. Without a dominant task set imposing its will, a whole host of cell assemblies are weakly activated. Even if one of them gains enough traction to rise to working memory, it's impossible for this coalition to install itself as the next powerful dictator for the simple reason that the costly inhibition processes necessary to suppress all other would-be dictators are not available in a mental state marked by hypofrontality. So its reign is short-lived.

Task set inertia guarantees that the old, deposed task set still has some strength. This is likely to be stronger in daydreaming because the ousting is followed by a power 
vacuum. As long as the ensuing succession conflict fails to bring forth a new ruler there is more room for diversity and different voices among the brain's many independent units. Recall also that the goal state of the overthrown task set remains activated - but weakly and in the fringe of working memory. This representation would not have the same quality and complexity it had in center stage but it would still contain a fitness function (a goal representation) that acts as a strange attractor for the trial-and-error operations of the search process in the underlying knowledge structure. The network then effectively runs simulations below the threshold of consciousness that hypothesis-test the different kinds of solutions to the problem - a variation process, perhaps - by assessing their speed of processing - the selection process. Should one mutant ideational combination be processed by the network at very high speeds, it might strengthen the internal stability of the task set enough to catapult its representation (a possible solution) into the working memory buffer. And by such means do insights occur.

\section{A funny thing happened on the way to the forum}

Without a global task set playing dictator, several different, but weakly-interacting task sets jockey for position, many taking brief turns under the sword of Damocles. Suppose now that an unexpected event occurs, say, you hop into the bathtub and displace a lot of water or you witness an apple fall from a tree. Actually, it doesn't even have to be a real event. It could be, for the sake of illustration, that a neuronal coalition still processes, in TSI style, bits of the TV documentary you saw yesterday about wild Australia and, as part of this memory reconstruction, happens to be mentally representing, oh, say, a kangaroo. Serendipitous events like these can produce interference ripples in the fading task set that still busies itself with searching the solution space of the puzzle problem. As the spreading activation now interacts with kangaroo or falling-apple knowledge nodes, new ideational combinations become possible. If one of them exceeds some fitness criteria held as a goal representation in fringe working memory, it is processed with higher speeds, crosses the oscillatory strength threshold needed for consciousness and, voilá, we experience an insight.

The difference to the deliberate mode is that the above scenario is unlikely under the oppressive thumb of one dominant task set that strongly directs the search and recombinatorial processes in the knowledge structure. For a start, the task set holding sway would actively inhibit exactly these sorts of carryover or ripple effects for the obvious reason that they pose a threat to its rule. What's more, the supreme task set inhibits not only other task sets but also filters the incoming sensory information falling apples, slow elevators, etc - and puts most of them into the category of 'irrelevant'. Largely this is because the leading coalition embodying that task set cannot recognize the meaning of the unexpected event due to its lopsided weight assignment. However, with a multitude of different task sets weakly activated, as is the case in daydreaming, the odds are better that one of them can assign meaning to the unanticipated event. And how would the network recognize what is meaningful, I 
hear you ask? The answer is simpler than you think: Speed of processing! Adding a few new information bits to the spreading activation might connect the dots in such a way as to make the processing in that network go smoother. You could say that altered mindsets, like dreaming, daydreaming, meditation or physical exercise facilitate certain types of insights (notice the careful phrasing) because they delude the task set. The hypofrontality process in such mental states makes it unlikely that strong top-down inhibition processes, which are required for a strong government, can be maintained.

It goes without saying that the strength of a neuronal coalition holding a task set is determined by how long and how hard you have thought about the puzzle problem. A well-learned, well-practiced, often considered task, leads to higher inertia once you start the incubation phase. In addition, the re-ignition of such a well-trotted network is more readily triggered by sensory input, even if the incoming information seems irrelevant. On the flipside, this can go too far when, for instance, everything you see reminds you of that puzzle problem. But such obsessiveness can be very useful during incubation considering the very fortuitous combination of strong TSI coupled with weak top-down biases imposed by prefrontal-induced search heuristics. Henry Poincaré (1921) spoke from experience when he said: 'Chance favors only the prepared mind.'

\section{The prepared mind}

Incubation enhances, sometimes, certain types of creativity because several parameters governing the network that computes novel information change as a function of interactions between it and other neural networks. To say that incubation enhances creativity is, unqualified like this, wrong outright. Whether incubation works depends mostly, it seems, on where the solution is located in design space, which of course, we don't know ahead of time. If located in the direction of common wisdom, deliberate thinking is the more creative mode of processing; if orthogonal to it, the spontaneous mode has a higher chance to hit upon the right move.

Since we have no direct access into the operations in our brains, the experience of sudden understanding without intentional reasoning is so discombobulating to us that it isn't surprising that such insights are often described with mystifying metaphors like being hit by a ton of bricks. But just because the steps leading to a revelation escape all efforts of introspective detection does not mean that there aren't any steps, that is. The variation-selection ratchet still churns over. As a result of many generate-and-test trials, with each iteration being an improvement on the speed of processing in the knowledge structure, the quality crank of the evolutionary algorithm running on the network can eventually lead to a complete restructuring of the problem space, and thus the puzzle's resolution. All we experience, however, is the final product, the insight. This is scary because we get the feeling that there is a discontinuity in the thought process and thus a lack of direct control over the process on our part. But this feeling is simply due to the scaffold enabling the creative solution to fall, like a Wittgensteinian ladder, by the wayside instead of being also 
represented, alongside the solution, in the global workspace. But we better stop here. The ins-and-outs of mental scaffolding is a topic for another article.

\section{Works cited:}

Allport, A 1987 'Selection for action: Some behaviorial and neurophysiological considerations of attention and action', in H Heuer \& A F Sanders (eds), Perspectives on perception and action, Hillsdale, NJ: Lawrence Erlbaum, 395-419

Anderson, J R 1996 ‘ACT: A simple theory of complex cognition’ American psychologist 51, 355-65

Arden, R, Chavez, R C, Grazioplene, R \& Jung, R E 2010 'Neuroimaging creativity: A psychometric view' Behavioural brain research 214, 143-56

Baars, B J 1988 A cognitive theory of consciousness, Cambridge: Cambridge University Press.

Baddeley, A 2000 'The episodic buffer: a new component of working memory' Trends in cognitive sciences $4,417-23$

Blackmore, J S 1999 The meme machine, Oxford: Oxford University Press.

Cabeza, R \& Nyberg, L 2000 'Imaging cognition II: An empirical review of 275 PET and fMRI studies' Journal of cognitive neuroscience 12, 1-47

Collins, A M \& Loftus, E F 1975 'A spreading activation theory of semantic processing' Psychological review $82,407-28$

Churchland, P S 2002 Brain-wise: Studies in neurophilosophy, Cambridge, MA: MIT Press

Coleridge, S T 1816 Christabel, Kubla Khan, and the pains of sleep, London: John Murray

Cowan, N 2001 'The magical number 4 in short-term memory: A reconsideration of mental storage capacity' Behavioral and brain sciences 24, 87-185

Crick, F \& Koch, C 1998 'Consciousness and neuroscience’ Cerebral cortex 8, 97-107

Dehaene, S \& Naccache, L 2001 'Towards a cognitive science of consciousness: Basic evidence and a workspace framework' Cognition 79, 1-37

Dennett, D C 1991 Consciousness explained, Boston: Little, Brown \& Co

Dietrich, A 2003 'Functional neuroanatomy of altered states of consciousness. The transient hypofrontality hypothesis' Consciousness and cognition 12, 231-56

Dietrich, A 2004 'The cognitive neuroscience of creativity' Psychonomic bulletin and review 11, 101126

Dietrich, A 2007a Introduction to consciousness, London: Palgrave Macmillian

Dietrich, A 2007b 'Who's afraid of a cognitive neuroscience of creativity?' Methods 42, 22-7

Dietrich, A \& Kanso, R 2010 'A review of EEG, ERP and neuroimaging studies of creativity and insight' Psychological bulletin 136, 822-48

Dietrich, A, Taylor, J T \& Passmore, C E 2001 'AVP (4-8) improves concept learning in PFC damaged but not hippocampal-damaged rats' Brain research 919, 41-7

Dijksterhuis, A, \& Aarts, H 2010 'Goals, attention, and (un)consciousness' Annual review of psychology 61, 467-90

Gazzaniga, M S et al. 2009 Cognitive neuroscience, $3^{\text {rd }}$ ed, New York: WW Norton

Katz, A. 1997 'Creativity and the cerebral hemispheres', in M Runco (ed) Creativity research handbook, v.1, Cresskill, NJ: Hampton Press, 203-26

Kekulé, F A 1890 ‘Benzolfest: Rede’ Beriche der Deutschen Chemischen Gesellschaft 23, 1302-11 
Koriat, A 2000 'The feeling of knowing: Some metatheoretical implications for consciousness and control' Consciousness and cognition 9, 149-71

Monsell, S 2003 'Task switching' Trends on cognitive science 7, 134-40

Reber, A S 1993 Implicit learning and tacit knowledge, Oxford: Oxford University Press

Runco, M 2004 'Creativity’ Annual review of psychology 55, 657-87

Poincaré, H 1921 The foundation of science: Science and hypothesis, the value of science, and science and method, New York: Science Press

Schultz, W 2000 'Multiple reward systems in the brain; Nature neuroscience 1, 199-207

Selfridge, O 1959 'Pandemonium: A paradigm for learning', in Proceedings of the Symposium on the mechanization of thought processes held at the National Physics Laboratory, November 1958, London: HM Stationary Office

Tulving, E 1995 'Organization of memory: Quo vadis?', in M S Gazzaniga (ed), The cognitive neurosciences, Cambridge: MA: MIT Press, 839-47

Waszak, F, Hommel, B, \& Allport, A 2003 'Task-switching and long-term priming: Role of episodic stimulus-task bindings in task-shift costs' Cognitive psychology 46, 361-413 